\title{
Significant progression of load on the musculoskeletal system with extremely high loads, with rapid weekly weight gains, using the Anatoly Gravitational System, in a I 0-week training period [Corrigendum]
}

\author{
Burke DT, Tran D, Cui D, et al. Open Access J Sports Med. \\ 2013;4:211-219.
}

On page 212, second paragraph of the Methods section, line 3 from the bottom, " $45 \mathrm{~kg}$ " should be " $30 \%$ ".

On page 213, first column, lines 9, 22 and 34, " $45 \mathrm{~kg}$ " should be " $30 \%$ ".

Table 1 on page 214, data entered into the Chest column under Maximum loads lifted, $\mathrm{kg}$ was incorrect. The corrected table is shown below.
On page 214, Belt-lifting system section, line 3, "297 kg" should be " $271 \mathrm{~kg}$ ".

On page 215, Chest press section, line 4, " 519.09 kg" should be " $257 \mathrm{~kg}$ ".

On page 215, Leg press section, line 2, " $377.27 \mathrm{~kg}$ " should be "376 kg".

On page 216 , first column, line 1 , " $423.05 \mathrm{~kg}$ " should be “422 kg”.

Table I Summary of loads lifted using the Anatoly Gravitational System during a I0-week training period

\begin{tabular}{|c|c|c|c|c|c|c|c|c|c|c|c|c|}
\hline & \multicolumn{4}{|c|}{ Mean loads lifted, kg } & \multicolumn{4}{|c|}{ Minimum loads lifted, kg } & \multicolumn{4}{|c|}{ Maximum loads lifted, kg } \\
\hline & Belt & Chest & Hand & Leg & Belt & Chest & Hand & Leg & Belt & Chest & Hand & Leg \\
\hline Session I & 271 & 110 & 115 & 256 & $|4|$ & 50 & 55 & $|4|$ & 447 & 187 & 224 & 376 \\
\hline Session 2 & 323 & 124 & 135 & 292 & 164 & 60 & 55 & 164 & 518 & 210 & 268 & 455 \\
\hline Session 3 & 369 & 136 & $|5|$ & 323 & 188 & 66 & 69 & 211 & 565 & 201 & $27 \mid$ & 534 \\
\hline Session 4 & 408 & 145 & 166 & 347 & 211 & 69 & 79 & 235 & 636 & 210 & 295 & 518 \\
\hline Session 5 & 442 & 152 & 177 & 367 & 235 & 69 & 79 & 245 & 660 & 224 & 318 & 499 \\
\hline Session 6 & 472 & 156 & 184 & 382 & 259 & 256 & 79 & 259 & 707 & 234 & 295 & 494 \\
\hline Session 7 & 498 & 160 & 189 & 395 & 282 & 69 & 79 & 269 & 730 & 234 & 295 & 542 \\
\hline Session 8 & 519 & 162 & 196 & 407 & 306 & 69 & 84 & 282 & 754 & 234 & 318 & 542 \\
\hline Session 9 & 539 & 166 & 200 & 414 & 329 & 74 & 93 & 292 & 786 & 248 & 318 & 542 \\
\hline Session 10 & 556 & 167 & 204 & 422 & 329 & 79 & 93 & 292 & 786 & 257 & 328 & 542 \\
\hline
\end{tabular}

\section{Publish your work in this journal}

Open Access Journal of Sports Medicine is an international, peer-reviewed, open access journal publishing original research, reports, reviews and commentaries on all areas of sports medicine. The manuscript management system is completely online and includes a very quick and fair peer-review system.
Visit http://www.dovepress.com/testimonials.php to read real quotes from published authors. 